\title{
A Combination of PD Controller and PIAFC for Stabilization of "x" Configuration Quadcopter
}

\author{
Ni'am Tamami,EndraPitowarno, I Gede Puja Astawa \\ Electronic Engineering Polytechnic Institute of Surabaya \\ Kampus PENS, Jalan Raya ITS, Sukolilo, Surabaya 60111, INDONESIA \\ E-mail: \{niam,epit,puja\}@pens.ac.id
}

\begin{abstract}
This paper presents a stabilization control method for " $\mathrm{x}$ " configuration quadcopter. The control method used the combination of PD (Proportional Derivative) controller and PIAFC (Proportional Integral Active Force Control). PD is used to stabilize quadcopter, and PIAFC is used to reject uncertainty disturbance (e.g. wind) by estimating disturbance torque value of quadcopter. The PD with PIAFC provided better result where PIAFC could minimize uncertain disturbance effect. The simulation has successfully give comparation about controller performance (PD, PD-AFC, PD-PIAFC) by calculate RMS (Root Mean Square) value. PD with AFC gives better result than PD. AFC optimization using PI (PD-PIAFC) give best result if compared with PD or PD-AFC. PD-PIAFC has lowest RMS value of result control signal, 0.0389 for constant disturbance and 0.1008 for fluctuated disturbance.
\end{abstract}

Keywords: "x" configuration quadcopter, stability, PD, PIAFC.

\section{INTRODUCTION}

Multicopter, a type of UAV is popular among hobbyist and researchers. It has advantagesin thrust, fast maneuver and VTOL ability (Vertical Take Off Landing). Multicopter has motors located at each end of the frame to produce greater thrust than other UAV in same size. Muticopter has a good handling even in a narrow space due to its fast response maneuverability. During development, multicopter able to turn into high speedUAV by altering the orientation and control techniques on quadcopter (quadshoot) that developed by Massachusetts Institute of Technology. VTOL capability facilitates multicopter for taking off and landing on a narrow area. In addition, the quadcopter is an interesting research material among researchers for many novelties that can be applied, for example in the control system, navigation techniques, real-time systems and robotics.

This paper focuses on quadcopter. Quadcopter has a simple structure. It utilizes rotors which are directed upwards and placed at the end of a 
crossedframe.The quadcopter is controlled by adjusting the rotation speed of each rotor. This research use " $x$ " configuration quadcopter because this configuration is considered more stable than "+" quadcopter configuration [1]. The " $x$ " configuration quadcopter has a better distribution of rotor forcesduring flying. The " $\mathrm{x}$ " quadcopter configuration structure can be found in Section 2.

Quadcopter is excellent object for investigating issues in control science. There are many research about quadcopter control algorithm and uncertainty disturbance rejection. Bouabdallah et al. designed an LQ controller and PID controller then compare it [2]. The result PID controller better than LQ controller. Jun Li and Yuntang Li designed PID controller to control angular and linear position, and succes to stabilize quadcopter [3].Mokhtari and Benallegue applied state parameter control to quadcopter rotation angle [4].By using state observer, quadcopter can measure external disturbance. Bora and Erdinc have been controlling position of quadcopter using PD controller and combined by using vision system [5]. Pounds et al. developed independent linear SISO controllers to regulate quadcopter using PID controller [6]. Inkyu Sa and Peter Corke develop Verticle infrastructure inspection using a quadcopter and shared autonomy control using PID method and successful stabilize quadcopter [7]. Sumantri et al. designed a sliding mode control using a nonlinear sliding surface (NSS) to design a robust tracking controller for a quad-rotor helicopter [8]. Chen and Huzmezan used linear $\mathrm{H} \infty$ controller to achieve stabilization in angular rates, vertical velocity, longitudinal velocity, lateral velocity, yaw angle and height of a quadcopter [9]. A linear $\mathrm{H} \infty$ controller can be designed to obtain stabilization and tracking performance using a systematical approach [10]. Pitowarno have designed Active Force Control and Knowledge-Based System for planar two-joint robot arm to improve performance of Active Force Control [11]. Katsura et al. have been modeled force sensing using disturbance observer without force sensor [12]. Chen et al. designed disturbance observer control for non linear system to control robotic manipulator [13]. Achtelik et al. has develop quadcopter using Model Reference Adaptive Control to reject uncertainty disturbance. Controller has been successful stabilize quadcopter [14].

It is very important to make a simple control algorithm to control the quadcopter stability although get uncertainty disturbance from environment, because in real system control algorithm will be embed in low speed data processing unit. PD can stabilize quadcopter but not good enough to maintain the quadcopter against uncertainty disturbance such as wind. PIAFC has the ability to estimate the force on the system without using complicated mathematical computation.

The purpose of this work is modelling and combining PD and PIAFC to control " $\mathrm{x}$ " configurationquadcopter when hover even if get uncertainty disturbance.This paper is structured as follows. Section 2, presents a quadcopter dinamic modelling. Section 3, deals quadcopter controller design. 
Section 4, presents the performance of the controller is shown in numerical simulations. Finally, in Section 5 conclusions of this work.

\section{QUADCOPTER MODELLING}

In this section the mathematical model of the quadcopter will be presented. This dynamic model contain the model of the rotor force and torque, gyroscopic effect, and the derrived force model of " $\mathrm{x}$ " configuration quadcopter.

Figure 1 is the design of " $\mathrm{x}$ " configuration quadcopter. The rotors (M1, M2, M3, M4) are placed in sequence $\pi / 4,3 \pi / 4,5 \pi / 4,7 \pi / 4$. Two diagonal rotors (M1 and M3) are rotating in the same direction (counter clockwise) whereas the others (M2 and M4) in the clockwise direction to eliminate the anti-torque that caused by rotor rotation.

Absolute position of the quadcopter can be described by a coordinate position of the body frame $\{B\}$ with reference earth frame $\{E\}$. Absolute attitude of the quadcopter can be described by three Euler's angles, $(\phi, \theta, \psi)$, which are roll, pitch and yaw with reference body frame $\{B\}$ when $X B, Y B$ and $\mathrm{ZB}$ axis in parallel with $\mathrm{X}, \mathrm{Y}$ and rotating 180 degree of $\mathrm{Z}$ axis.

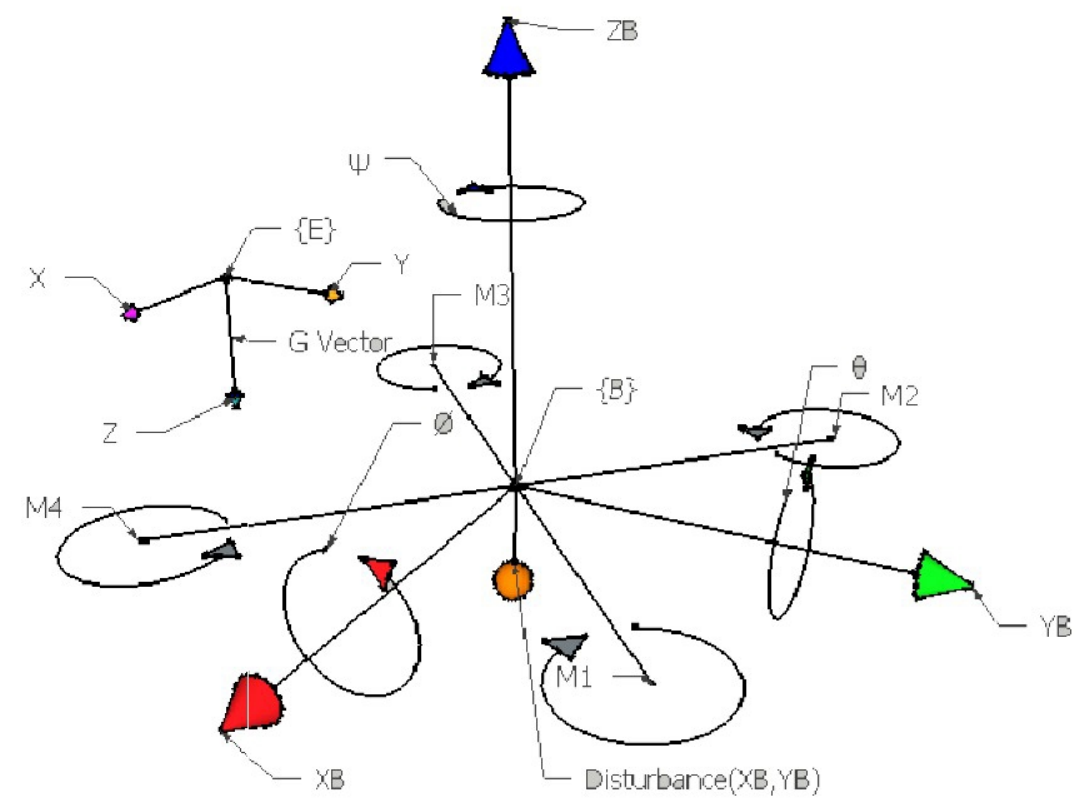

Figure 1.An " $\mathrm{x}$ " configuration quadcopter

To make a movement along $\mathrm{XB}$ axis, quadcopter must produce pitch torque $\left(\tau_{y}\right)$. It means, quadcopter decreases rotor speed at M1 and M4, and increase rotor speed at M2 and M3. Likewise to make movement along YB axis, quadcopter must produce roll torque $\left(\tau_{x}\right)$. Quadcopter decreases rotor speed at M1 and M2, and increase rotor speed at M3 and M4. Then, to change 
quadcopter heading, quadcopter must produceyaw torque ( $\tau_{z}$ ) by increasing M1 and M3 rotor speed, and decreasing M2 and M4 rotor speed.

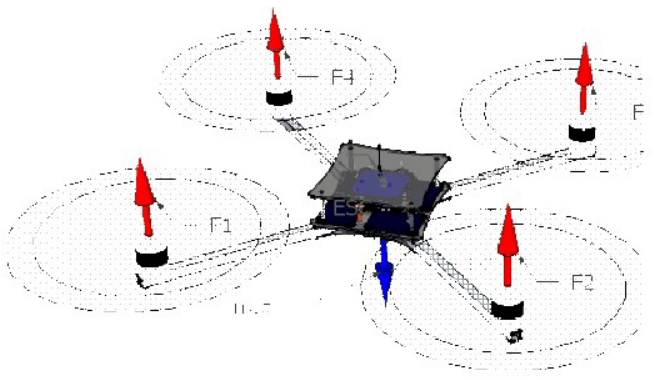

Figure 2. Forcedistribution in quadcopter

Figure 2shows the force distribution in quadcopter. "F1, F2, F3, F4" arrows are thrust force each motor, and "m.g" arrow is weight force of quadrotor. From Li et al., the thrust and hub force for each rotor $\left(F_{i}, H_{i}\right)$ can be represented in (1)and (2) [3].Thrust force is the resultant of the vertical forces acting on all the blade elements. Hub force is the resultant of the horizontal forces acting on all the blade elements. Where $\rho$ is air density. $C_{T}$ is thrust constant that depend on polar lift slope, geometric blade, velocity trough motor, the ratio the surface area and rotor disk area [6]. $C_{d}$ is drag constant, and $\Omega_{i}$ is propeller rotation speed.

$$
\begin{aligned}
F_{i} & =0.5 \rho C_{T} \Omega_{i}^{2} \\
& =k_{t} \Omega_{i}^{2} \\
H_{i} & =\rho C_{d} \Omega_{i}^{2} \\
& =k_{d} \Omega_{i}^{2}
\end{aligned}
$$

Quadcopter can change its position by combining translation and rotation angle. Linear movement on the quadcopter can be produced by total thrust force of the four rotor (3), whereas changes in the angle of rotation (roll, pitch, yaw) will cause a change in the direction of translational movement quadcopter. Total force of the quadcopter can be decomposed into force element in each axis $\left(F_{x}, F_{y}, F_{z}\right)$. Figure 3 shows the illustration of force decomposition to each axis in body frame $\{B\}$.

$$
F_{\text {total }}=\sum_{i=1}^{4} F_{i}
$$




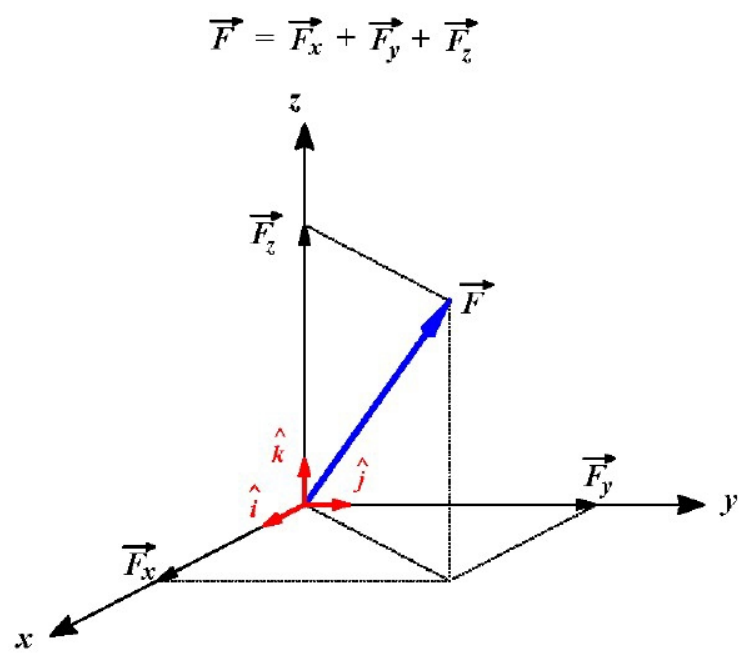

Figure 3. Total forces illustration that decomposed into each axis

Equation (4) is rotation matrix of quadcopter. $C, S$ are cosine function and sine function respectively.

$$
R=\left[\begin{array}{ccc}
C \phi C \theta & C \phi S \theta_{s} \psi-C \psi S \phi & S \phi S \psi+C \phi C \psi S \phi \\
C \theta S \phi & C \phi C \psi+S \phi S \theta S \psi & C \psi S \phi C \theta-C \phi S \psi \\
-S \theta & C \theta S \psi & C \theta C \psi
\end{array}\right]
$$

The derrived model of quadcopter translational movement can be represented as (5). Where $\ddot{x}, \ddot{y}, \ddot{z}$ are linear acceleration in of quadcopter in each axis.

$$
m\left[\begin{array}{c}
\ddot{x} \\
\ddot{y} \\
\ddot{z}
\end{array}\right]=R\left[\begin{array}{c}
0 \\
0 \\
F_{\text {total }}
\end{array}\right]-\left[\begin{array}{c}
0 \\
0 \\
m g
\end{array}\right]
$$

The model also contain gyroscopic effect. Derrived torque model of quadcopter are presented at (6), (7), (8).

$$
\tau_{x}=\left[\begin{array}{l}
F_{1} \\
F_{2} \\
F_{3} \\
F_{4}
\end{array}\right]^{T} l\left[\begin{array}{c}
C\left(\frac{\pi}{4}\right) \\
C\left(\frac{3 \pi}{4}\right) \\
C\left(\frac{5 \pi}{4}\right) \\
C\left(\frac{7 \pi}{4}\right)
\end{array}\right]+\dot{\theta} \dot{\psi}\left(I_{y y}-I_{z z}\right)
$$




$$
\begin{gathered}
\tau_{y}=\left[\begin{array}{l}
F_{1} \\
F_{2} \\
F_{3} \\
F_{4}
\end{array}\right]^{T} l\left[\begin{array}{c}
S\left(\frac{\pi}{4}\right) \\
S\left(\frac{3 \pi}{4}\right) \\
S\left(\frac{5 \pi}{4}\right) \\
S\left(\frac{7 \pi}{4}\right)
\end{array}\right]+\dot{\phi} \dot{\psi}\left(I_{z z}-I_{x x}\right) \\
\tau_{z}=K_{d} l\left(-F_{1}+F_{2}-F_{3}+F_{4}\right)+\dot{\theta} \dot{\phi}\left(I_{x x}-I_{y y}\right)
\end{gathered}
$$

$\tau_{x}, \tau_{y}, \tau_{z}$ are roll, pitch, yaw torque respectively. $l$ is distance of rotor between center of mass. $\dot{\phi}, \dot{\theta}, \dot{\psi}$ are roll, pitch, yaw angular body speed respectively. $I_{x x}, I_{y y}, I_{z}$ are roll, pitch, yaw body inertia respectively. $K_{d}$ is force resistance constant in (2).

Let us define the control inputs of quadcopter are $u_{1}, u_{2}, u_{3}, u_{4}$. Where $u_{1}$ is total force control input. Total force control input can derrived by subtitute (1) to (3). $u_{2}$ is roll torque control input, $u_{3}$ is pitch torque control input, and $u_{4}$ is yaw torque control input can derrived by subtitute (1) to (6), (7) and (2) to (8). Where, $k_{t}$ and $k_{d}$ are constant value from (1), (2).

$$
\begin{aligned}
u_{1} & =F_{\text {total }} \\
& =k_{t} \sum_{i=1}^{4} \Omega_{i}^{2} \\
u_{2} & =\tau_{x} \\
& =k_{t} l \sum_{i=1}^{4} \Omega_{i}^{2} \cos \left(\frac{\pi}{2}(i-1)+\frac{\pi}{4}\right) \\
u_{3} & =\tau_{y} \\
& =k_{t} l \sum_{i=1}^{4} \Omega_{i}^{2} \sin \left(\frac{\pi}{2}(i-1)+\frac{\pi}{4}\right) \\
u_{4} & =\tau_{z} \\
& =k_{d} l \sum_{i=1}^{4}(-1)^{i} \Omega_{i}^{2}
\end{aligned}
$$

By subtitute (9) into (5), (6), (7), (8), the derrived model of quadcopter in (10). Where $\ddot{\phi}, \ddot{\theta}, \ddot{\psi}$ are roll, pitch, yaw, angular acceleration at quadcopter body. 


$$
\begin{aligned}
& \ddot{x}=\frac{(s \phi s \psi+c \phi c \psi s \theta) u_{1}}{m} \\
& \ddot{y}=\frac{(c \psi s \phi c \theta-c \phi s \psi) u_{1}}{m} \\
& \ddot{z}=\frac{(c \theta s \psi) u_{1}}{m}-g \\
& \dot{\phi}=\frac{u_{2}+\dot{\theta} \dot{\psi}\left(I_{y y}-I_{z z}\right)}{I_{x x}} \\
& \ddot{\theta}=\frac{u_{3}+\dot{\phi} \dot{\psi}\left(I_{z z}-I_{x x}\right)}{I_{y y}} \\
& \dot{\psi}=\frac{u_{4}+\dot{\theta} \dot{\phi}\left(I_{x x}-I_{y y}\right)}{I_{z z}}
\end{aligned}
$$

Mechanical energy there are kinetic energy $\left(E_{K}\right)$ and potential energy( $E_{P}$ ) in quadcopter can define as :

$$
\begin{aligned}
& E_{K}=0.5 I_{x x}(\dot{\phi}-\dot{\psi} s \theta)+0.5 I_{y y}(\dot{\theta} c \phi+c \theta s \phi)^{2}+0.5 I_{z z}(\dot{\theta} s \phi-\dot{\psi} c \theta c \phi)^{2} \\
& =0.5 I_{x x} \dot{\phi}^{2}+0.5 I_{y y} \dot{\theta}^{2}+0.5 I_{z z} \dot{\psi}^{2} \\
& E_{P}=\int x d m(x) \cdot(-g s \theta)+\int y d m(y) \cdot(c \theta s \phi)+\int z d m(z) \cdot(c \phi c \theta)
\end{aligned}
$$

\section{QUADCOPTER CONTROLLER DESIGN}

In this section, the control algorithm of quadcopter is presented. The purpose is tocombine PD and PIAFC as rotational controller to stabilize quadcopter. Figure 4shows the proposed rotational controller to stabilize quadcopter.

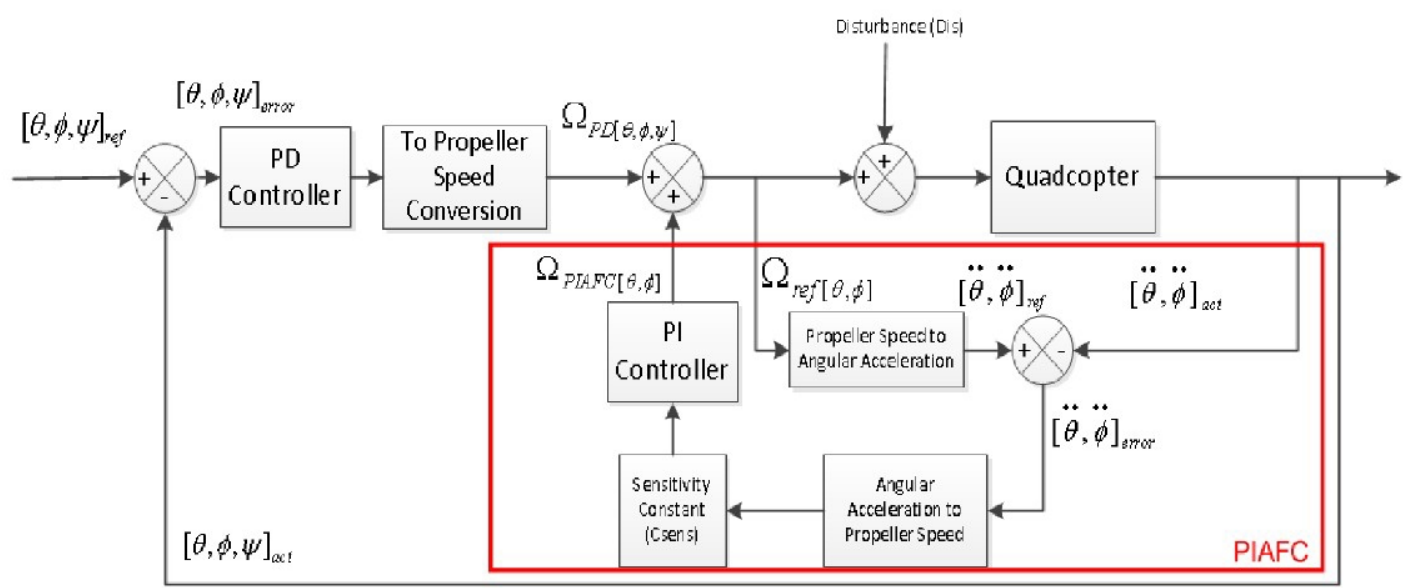

Figure 4.The proposed rotational controller 
Figure 5 shows quadcopter control structure. In this simulation, translational movement are neglected. The controller design is focused to stabilize the quadcopter toward disturbance. PD controller is used to stabilize quadcopter and PIAFC to reject uncertainty disturbance from environment. In this simulation, quadcopter get constant and fluctuated disturbance.

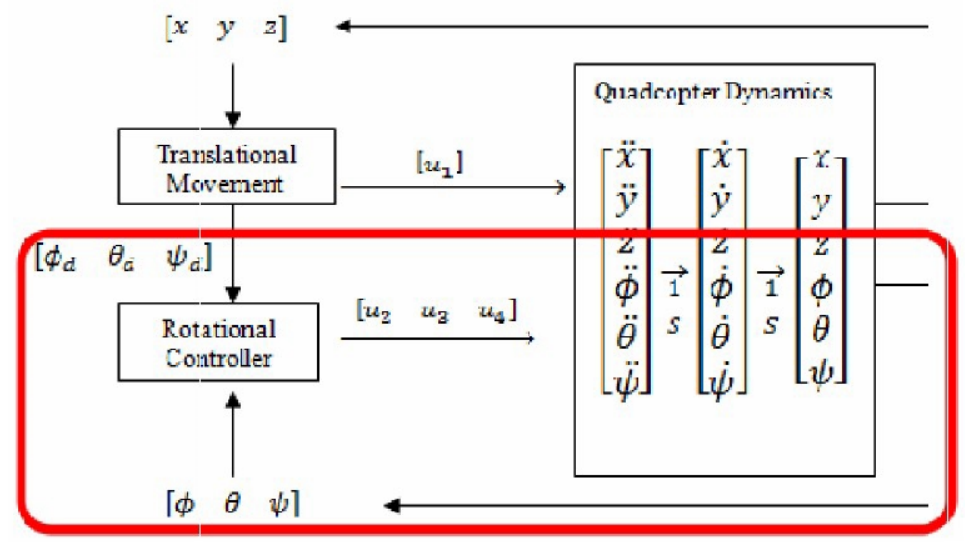

Figure 5.Quadcopter control structure

FromFigure 5, the relationship of each input and each state can be represented as (13).

$$
\begin{aligned}
\dot{X} & =A X+B U \\
X & =\left[\begin{array}{llllll}
\phi & \theta & \psi & \dot{\phi} & \dot{\theta} & \dot{\psi}
\end{array}\right]^{T} \\
U & =\left[\begin{array}{llllll}
u_{1} & u_{2} & u_{3} & u_{4}
\end{array}\right] \\
X & =\left[\begin{array}{llllll}
\dot{\phi} & \dot{\theta} & \dot{\psi} & \ddot{\phi} & \ddot{\theta} & \ddot{\psi}
\end{array}\right]^{T}
\end{aligned}
$$

The system matrix (A) can be represented as (14).

$$
A=\left[\begin{array}{cccccc}
0 & 0 & 0 & 1 & 0 & 0 \\
0 & 0 & 0 & 0 & 1 & 0 \\
0 & 0 & 0 & 0 & 0 & 1 \\
0 & 0 & 0 & 0 & \frac{\dot{\psi}\left(I_{y y}-I_{x x}\right)}{2 I_{x x}} & \frac{\dot{\theta}\left(I_{y y}-I_{x x}\right)}{2 I_{x x}} \\
0 & 0 & 0 & \frac{\dot{\psi}\left(I_{z z}-I_{x x}\right)}{2 I_{y y}} & 0 & \frac{\dot{\phi}\left(I_{z z}-I_{x x}\right)}{2 I_{y y}} \\
0 & 0 & 0 & \frac{\dot{\theta}\left(I_{x x}-I_{y y}\right)}{2 I_{z z}} & \frac{\dot{\phi}\left(I_{x x}-I_{y y}\right)}{2 I_{z z}} & 0
\end{array}\right]
$$

The control matrix (B) can be represented as (15). 


$$
B=\left[\begin{array}{cccc}
0 & 0 & 0 & 0 \\
0 & 0 & 0 & 0 \\
0 & 0 & 0 & 0 \\
0 & \frac{1}{I_{x x}} & 0 & 0 \\
0 & 0 & \frac{1}{I_{y y}} & 0 \\
0 & 0 & 0 & \frac{1}{I_{z z}}
\end{array}\right]
$$

\subsection{Disturbance Model}

In this subsection, the model of disturbance will be presented. The disturbance vector(Dis) that used in this paper consists of two types. First simulation use constant disturbance ( $D i s_{C X}$ ), and second simulation fluctuated/uncertainty disturbance ( $D i s_{F X}$ ). Figure 1 shows disturbance position of quadcopter, disturbance mass located at ( $\mathrm{L}_{\mathrm{DXB}}, \mathrm{L}_{\mathrm{DYB}}$ ) from the center of quadcopter in (XB, YB) axis. By including disturbance vector, state equation (13) can be written as follows:

$$
\dot{X}=A X+B U+D i s
$$

First simulation with constant disturbance:

$$
\text { Dis }=\left[\begin{array}{llllllll}
0 & 0 & 0 & D i s_{C X} & L_{D Y B} & D i s_{C X} & L_{D X B} & 0
\end{array}\right]^{T}
$$

Second simulation with fluctuated disturbance:

$$
\text { Dis }=\left[\begin{array}{llllllll}
0 & 0 & 0 & D_{i s_{F X}} & L_{D Y B} & D_{i s_{F X}} & L_{D X B} & 0
\end{array}\right]^{T}
$$

\subsection{PD Controller Design}

PD control algorithm is designed without disturbance parameter. The controller design is focused to stabilize quadcopter when hover without get uncertainty disturbance. The model that presented at Section 2 is completed by gyroscopic effect. If we design quadcopter stabilize at hover, it can be ignored because it dont have significant effect at quadcopter system [15]. The model can be simplified:

$$
\begin{aligned}
& \ddot{\phi}=\frac{k_{t} l \sum_{i=1}^{4} \Omega_{\mathrm{i}}{ }^{2} \cos \left(\frac{\pi}{2}(i-1)+\frac{\pi}{4}\right)}{I_{x x}} \\
& \ddot{\theta}=\frac{k_{t} l \sum_{i=1}^{4} \Omega_{\mathrm{i}}{ }^{2} \sin \left(\frac{\pi}{2}(i-1)+\frac{\pi}{4}\right)}{I_{y y}} \\
& \ddot{\psi}=\frac{k_{d} \sum_{i=1}^{4}(-1)^{i} \Omega_{\mathrm{i}}}{I_{z z}}
\end{aligned}
$$


The simulation purposes is to stabilize roll, pitch and yaw angle. By using twice integral operation at (19), the model can be rewritten as :

$$
\begin{aligned}
& \ddot{\phi}=\frac{k_{t} l \sum_{i=1}^{4} \Omega_{\mathrm{i}}^{2} \cos \left(\frac{\pi}{2}(i-1)+\frac{\pi}{4}\right)}{I_{x x} s^{2}} \\
& \ddot{\theta}=\frac{k_{t} l \sum_{i=1}^{4} \Omega_{\mathrm{i}}^{2} \sin \left(\frac{\pi}{2}(i-1)+\frac{\pi}{4}\right)}{I_{y y} s^{2}} \\
& \ddot{\psi}=\frac{k_{d} \sum_{i=1}^{4}(-1)^{i} \Omega_{\mathrm{i}}}{I_{z z} s^{2}}
\end{aligned}
$$

From (20), the model is second order form, therefore it can use classical controller to stabilize quadcopter. PD controller will be presented to stabilize quadcopter. The reason is this controller very simple and easy implemented. This is PD controller each orientation angle.

$$
u_{2}, u_{3}, u_{4}=P_{\phi, \theta, \psi}(\phi, \theta, \psi)+D_{\phi, \theta, \psi}(\phi, \theta, \psi)
$$

Where $u_{2}, u_{3}, u_{4}$ are control input for roll, pitch, yaw torque respectively. $P_{\phi, \theta, \psi}(\phi, \theta, \psi)$ are proporsional control for roll, pitch, and yaw respectively. $D_{\phi, \theta, \psi}(\phi, \theta, \psi)$ are derrivative control for roll, pitch, and yaw respectively.

\subsection{PIAFC Controller Design}

PIAFC controller is designed to reject uncertainty disturbance from environment. It is combined PI controller and AFC Controller. Figure 6 shows the PIAFC block diagram that used in simulation. This block has two inputs, first is measured angular velocity that differentiated into actual angular acceleration (22). Second input is applied propeller speed that converted into angular acceleration reference (24). To get estimated disturbance, actual angular acceleration is compared by angular acceleration reference (25). Last, convert the disturbance acceleration into propeller speed (26) then add the result with PD controller result. 


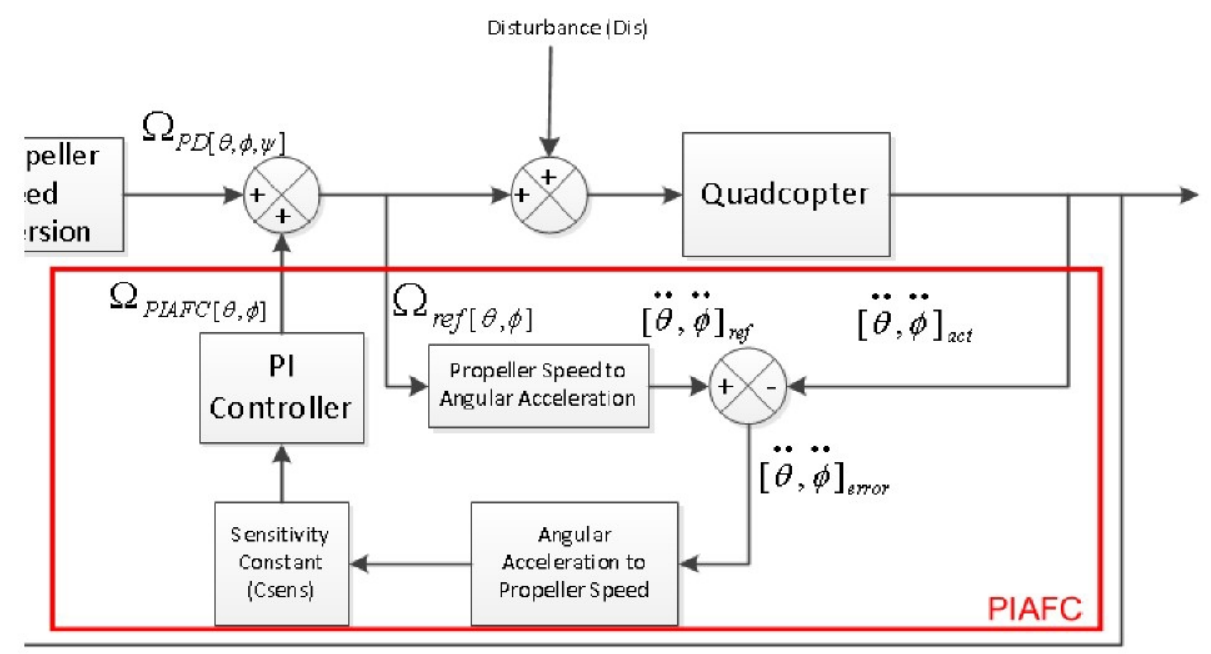

Figure 6.AFC block diagram

Let us define $\gamma$ is rotation angle roll and pitch axis $(\phi, \theta)$,

$$
\begin{aligned}
& \ddot{\gamma}=\frac{d \dot{\gamma}}{d t} \\
& \Omega_{\phi}=\Omega_{1}+\Omega_{2}-\Omega_{3}-\Omega_{4} \\
& \Omega_{\theta}=\Omega_{1}-\Omega_{2}-\Omega_{3}+\Omega_{4} \\
& \ddot{\gamma}_{r e f}=\frac{0.5 \rho C_{t} l \Omega_{\gamma}\left|\Omega_{\gamma}\right|}{I_{x x, y y}} \\
& \ddot{\gamma}_{A F C}=\ddot{\gamma}_{r e f}-\ddot{\gamma} \\
& \Omega_{P I A F C}=P\left(\ddot{\gamma}_{A F C}\right)+I\left(\ddot{\gamma}_{A F C}\right)
\end{aligned}
$$

$\ddot{\gamma}_{A F C}$ is disturbance angular acceleration estimation. $\Omega_{P I A F C}$ is propeller speed calculation form PIAFC controller output.

\subsection{Stability Analysis}

From (10), let us define angular equation motion within controller as,

$$
\begin{aligned}
& I_{x x} \ddot{\phi}=u_{2}+\dot{\theta} \dot{\psi}\left(I_{x x}-I_{z z}\right) \\
& I_{y y} \ddot{\theta}=u_{3}+\dot{\phi} \dot{\psi}\left(I_{z z}-I_{y y}\right)
\end{aligned}
$$

Because $u_{2}, u_{3}$ is input control, and the value of gyroscopic torque is very small, then the equation can be rewritten as,

$$
I_{x x} \ddot{\gamma}=P(\gamma)+D(\gamma)+P\left(\ddot{\gamma}_{A F C}\right)+I\left(\ddot{\gamma}_{A F C}\right)
$$


From (11), kinetic energy ( $\left.E_{K}\right)$ in XB axis as Lyapunov function (V),

$$
V=0.5 I_{x x, y y} \dot{\gamma}^{2}
$$

Then,

$$
\begin{aligned}
& \dot{V}=I_{x x, y y} \ddot{\gamma} \\
& \dot{V}=\left(K_{P}\left(\gamma_{r e f}-\gamma\right)+K_{D}\left(\gamma_{r e f}-\gamma\right)+K_{P I A F C}\left(\gamma_{A F C}-\gamma\right)\right) \dot{\gamma}
\end{aligned}
$$

Where $\left\lfloor\gamma_{\text {ref }}, \dot{\gamma}_{\text {ref }}, \ddot{\gamma}_{\text {ref }}\right\rfloor=[0,0,0]$, the equation can be rewritten as,

$$
\dot{V}=\left(-K_{P}(\gamma)-K_{D}(\dot{\gamma})+K_{P I A F C}(\ddot{\gamma})\right) \dot{\gamma}
$$

$\dot{V}$ is negative definite, and thus the stability of the closed loop dynamics in is guaranteed.

\section{SIMULATION AND ANALYSIS}

In this section, comparison result of PD, PD-AFC, and PD-PIAFC (AFC that optimized by PI controller) presented. Table 1 presents thequadcopter parameters that used in this simulation as follows are those for the experimental system in our laboratory.

Table 1. Quadcopter simulation parameter

\begin{tabular}{|l|l|l|}
\hline Parameter & \multicolumn{1}{|c|}{ Unit } & \multicolumn{1}{c|}{ Value } \\
\hline $\mathrm{m}$ & $\mathrm{Kg}$ & 1.025 \\
\hline $\mathrm{L}$ & Meter & 0.270 \\
\hline$k_{t}$ & $\mathrm{Ns}^{2}$ & $3.122 \mathrm{e}-06$ \\
\hline$k_{d}$ & $\mathrm{Nms}^{2}$ & $1.759 \mathrm{e}-08$ \\
\hline $\mathrm{Ixx}$ & $\mathrm{kgm}^{2}$ & 0.012 \\
\hline Iyy & $\mathrm{kgm}^{2}$ & 0.012 \\
\hline $\mathrm{Izz}$ & $\mathrm{kgm}^{2}$ & 0.048 \\
\hline Dis & Newton & 0.2 \\
\hline Dis & Newton & $0.2 S(2 \pi 0.4 t)$ \\
\hline $\mathrm{LDXB}_{\mathrm{DX}}$ & $\mathrm{mm}$ & 0 \\
\hline $\mathrm{L}_{\mathrm{DYB}}$ & $\mathrm{mm}$ & 190 \\
\hline
\end{tabular}

Table 2. PD coefficients simulation parameter

Note : * for PD, ${ }^{* *}$ for PIAFC.

\begin{tabular}{|l|c|}
\hline Parameter & Value \\
\hline $\mathrm{KP}^{*}$ & 0.097 \\
\hline $\mathrm{KD}^{*}$ & 0.036 \\
\hline $\mathrm{KP}^{* *}$ & 1.000 \\
\hline $\mathrm{KI}^{* *}$ & 0.100 \\
\hline
\end{tabular}


PD coefficients that used for simulation are derrived by trial and error to get best performance, the controller constant parameter are listed in Table 2. First simulation compares PD, PD-AFC, and PD-PIAFC performance if get constant disturbance. Second simulation compares PD, PD-AFC, and PDPIAFC performance if get fluctuated disturbance. Root Mean Square (RMS) method is used to determine the controller performance analysis. Lower RMS value means better performance of controller.
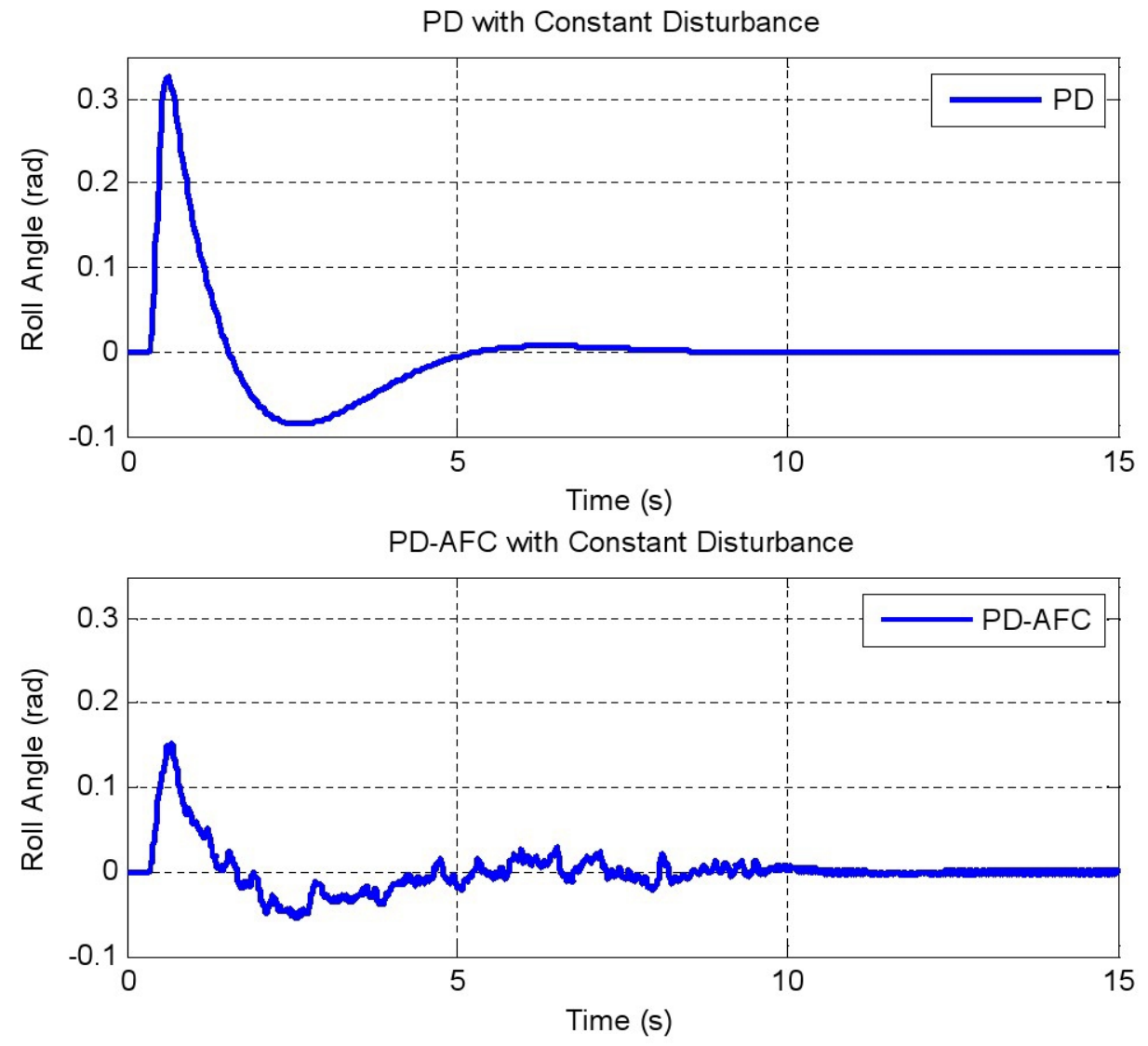

PD-PIAFC with Constant Disturbance

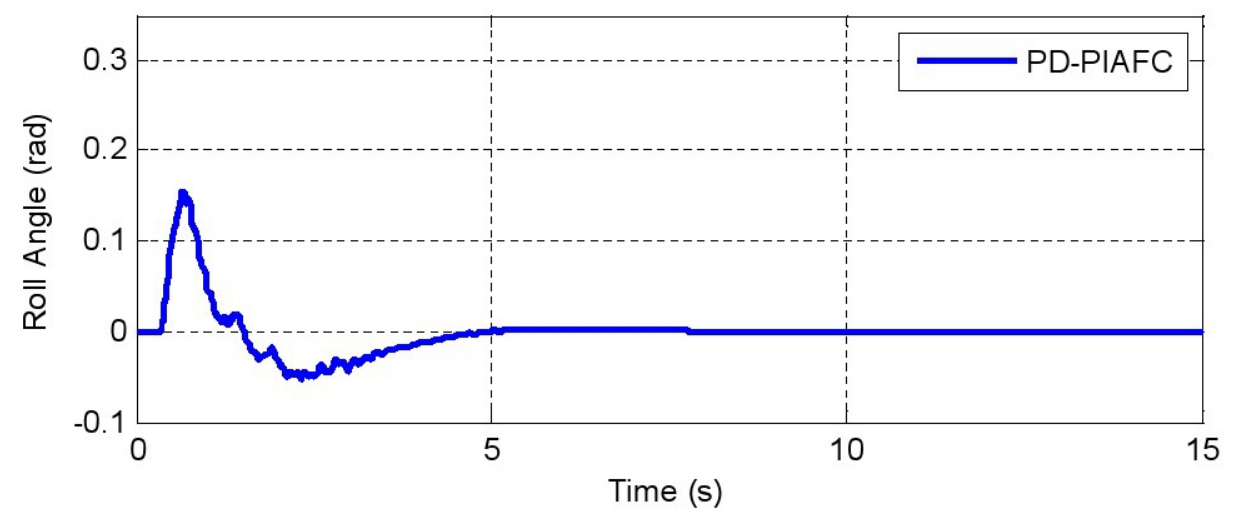

Figure 7.Constant disturbance simulation result 
Figure 7 shows the comparison result of PD, PD-AFC, and PD-PIAFC method with constant disturbance. By using PD, maximum error is 0.325 radian and can stable at 5 seconds. $\mathrm{PD}$-AFC and $\mathrm{PD}$-PIAFC give smaller error, 0.15 radian. $\mathrm{PD}-\mathrm{AFC}$ noise more than $\mathrm{PD}$-PIAFC. $\mathrm{PD}$ controller has RMS value 0.059 , PD-AFC is 0.041, and PD-PIAFC is 0.0389. From RMS value, we can conclude PD-PIAFC give best result of controller.

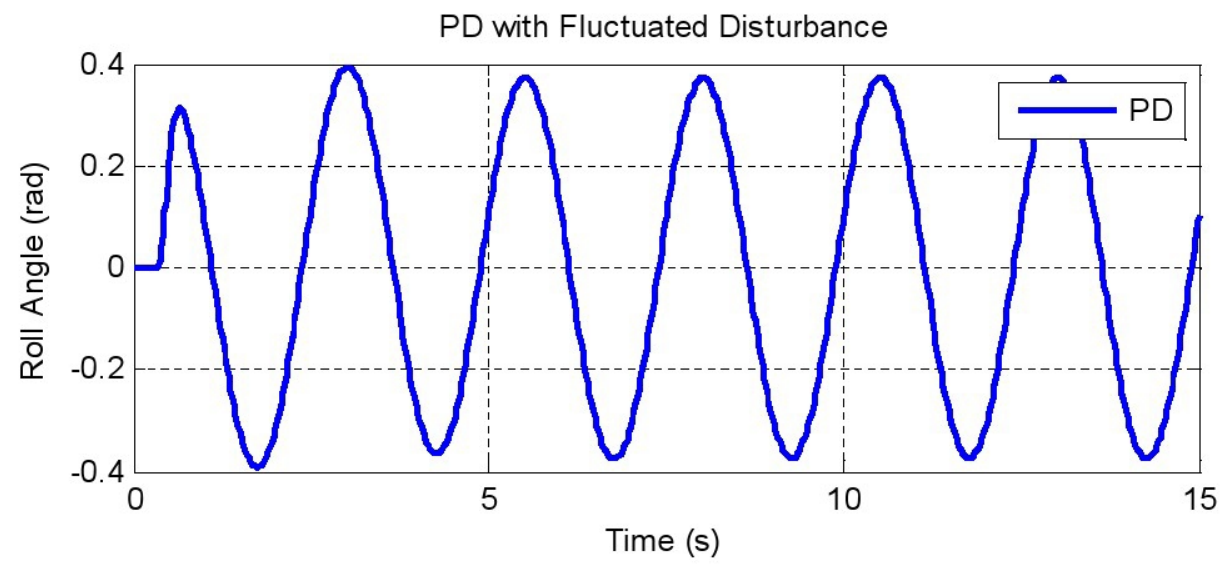

PD-AFC with Fluctuated Disturbance

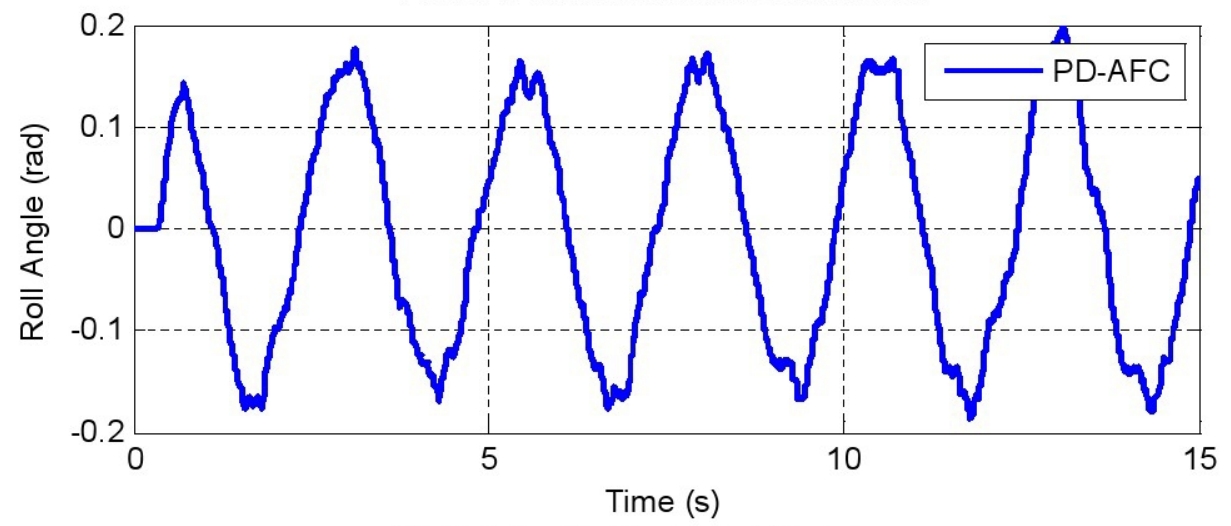

PD-PIAFC with Fluctuated Disturbance

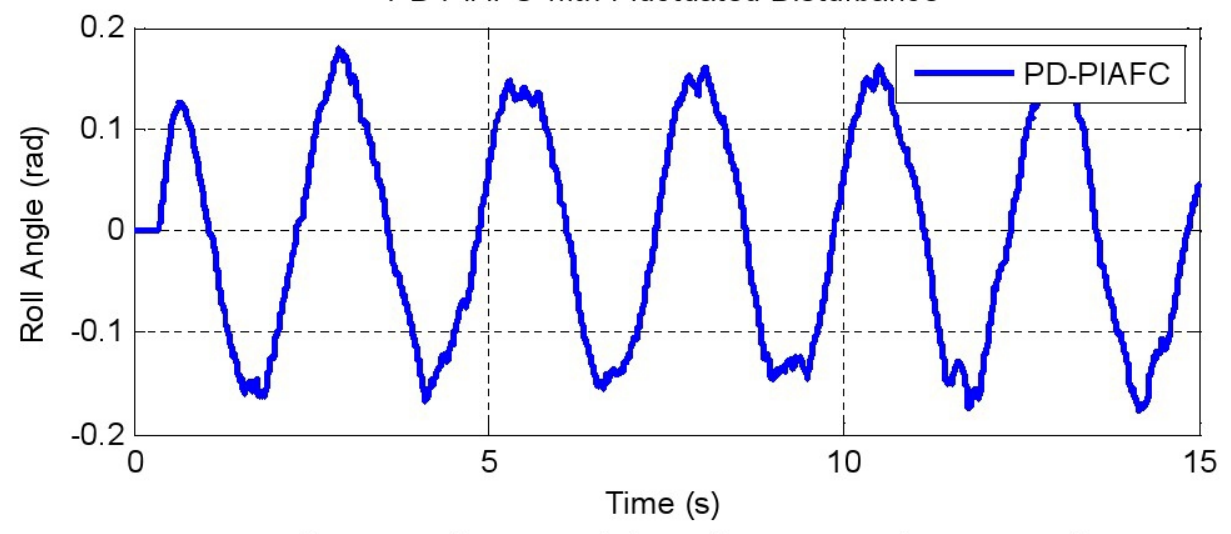

Figure 8.Fluctuated disturbance simulation result 
Figure 8 shows the simulation result of PD method, PD-AFC, and PDPIAFC method with fluctuated disturbance. In this simulation, PD maximum error is 0.4 radian. $\mathrm{PD}-\mathrm{AFC}$ and $\mathrm{PD}$-PIAFC give smaller error, 0.15 radian. $\mathrm{PD}-$ AFC noise more than PD-PIAFC. PD controller has RMS value 0.2586, PD-AFC is 0.1054 , and PD-PIAFC is 0.1008 . From RMS value, we can conclude PDPIAFC give best result of controller.

From the simulation, PIAFC controller can minimize the effect of disturbance. In each simulation, PD-PIAFC gives best result. PD-PIAFC has lowest RMS value, 0.0389 for constant disturbance and 0.1008 for fluctuated disturbance.

\section{CONCLUSION}

An "x" configuration quadcopter has been succesfully modeled. Then, simulation results have been presented to show the controller performance. The simulation has succesfull give comparation about controller performance (PD, PD-AFC, PD-PIAFC) by calculate Root Mean Square (RMS) value. PD with AFC gives better result than PD. AFC optimization using PI (PD-PIAFC) give best result if compared with PD or PD-AFC. PD-PIAFC has lowest RMS value of result control signal, 0.0389 for constant disturbance and 0.1008 for fluctuated disturbance.

\section{REFERENCES}

[1] Gupte, Shweta, Paul Infant Teenu Mohandas, and James M. Conrad,A Survey of Quadrotor Unmanned Aerial Vehicles, Proceedings of IEEE. IEEE, Southeastcon, pp. 1-6, 2012.

[2] Bouabdallah, Samir, Andre Noth, and Roland Siegwart, PID vs LQ Control Techniques Applied to an Indoor Micro Quadrotor.Intelligent Robots and Systems, 2004.(IROS 2004). Proceedings. 2004 IEEE/RSJ International Conference on. Vol. 3. IEEE, pp.2451-2456, 2004.

[3] Li, Jun, and Yuntang Li, Dynamic Analysis and PIDControl for aQuadrotor, Mechatronics and Automation (ICMA), 2011 International Conference on. IEEE, pp. 573-578, 2011.

[4] Mokhtari, Abdellah, and A. Benallegue, Dynamic Feedback Controller of Eulerangles and Wind Parameters Estimation for a Quadrotor Unmanned Aerial Vehicle, Robotics and Automation, 2004. Proceedings. ICRA'04. 2004 IEEE International Conference on. Vol. 3. IEEE, pp. 2359-2366, 2004.

[5] Erginer, Bora, and Erdinc Altug, Modeling and PD Control of a Quadrotor VTOL Vehicle,Intelligent Vehicles Symposium, 2007 IEEE. IEEE, pp. 894-899, 2007.

[6] Pounds, Paul, Robert Mahony, and Peter Corke, Modelling and Control of a Large Quadrotor Robot, Control Engineering Practice 18.7 Elsevier, 2010, pp. 691-699, 2010. 
[7] Sa, Inkyu, and Peter Corke,Vertical Infrastructure Inspection using a Quadcopter and Shared Autonomy Control, In Field and Service Robotics, pp. 219-232. Springer Berlin Heidelberg, 2014.

[8] Tayebi, A., and S. McGilvray, Attitude Stabilization of a Four-rotor Aerial Robot, Decision and Control, 2004. CDC. 43rd IEEE Conference on. Vol. 2. IEEE, pp. 1216-1221, 2004.

[9] Sumantri, Bambang, Naoki Uchiyama, Shigenori Sano, and Yuma Kawabata, Robust Tracking Control of a Quad-rotor Helicopter Utilizing Sliding Mode Control with a Nonlinear Sliding Surface, Journal of System Design and Dynamics 7, no. 2, pp. 226-241, 2013.

[10] Ming Chen and Mihai Huzmezan, A Combined MBPC/2DOF Ho Controller for a Quadrotor UAV, Proceeding of AIAA guidance, navigation, and control conference and exhibit, Texas- USA, 11-14 August 2003.

[11] Estiko Rijanto, Robust Control: Theory for Application, ISBN: 9799299-12-8, Bandung: ITB Press, 2000.

[12] Pitowarno, Endra, An Implementation of Acknowledge-based System Method to an Active Force Control Robotic Scheme, Master Thesis, Universiti Teknologi Malaysia, 2006.

[13] Katsura, Seiichiro, Yuichi Matsumoto, and Kouhei Ohnishi, Modeling of Force Sensing and Validation of Disturbance Observer for Force Control, Industrial Electronics, IEEE Transactions on 54, no. 1, pp. 530538, 2007.

[14] Chen, Wen-Hua, Disturbance Observer Based Control for Nonlinear Systems, Mechatronics, IEEE/ASME Transactions on 9, no. 4, pp.706710, 2004.

[15] Achtelik, Michael, Thomas Bierling, Jian Wang, Leonhard Höcht, and Florian Holzapfel, Adaptive Control of a Quadcopter in the Presence of Large/Complete Parameter Uncertainties, In Infotech@ Aerospace 2011, 2011.

[16] Bouabdallah, Samir, Pierpaolo Murrieri, and Roland Siegwart, Design and Control of an Indoor Micro Quadrotor, Robotics and Automation, 2004. Proceedings. ICRA'04. 2004 IEEE International Conference on. Vol. 5. IEEE, pp. 4393-4398, 2004.

[17] Benallegue, A., A. Mokhtari, and L. Fridman, Feedback Linearization and High Order Sliding Mode Observer for a Quadrotor UAV, Variable Structure Systems, 2006. VSS'06. International Workshop on. IEEE, pp. 365-372, 2006.

[18] Ni'am Tamami, Endra Pitowarno, I Gede Puja Astawa, Modelling and PD Control for " $\mathbf{x}$ " Configuration Quadcopter,Indonesian Symposium on Robot Soccer Competition, 2013. Proceedings. Dian Nuswantoro University. ISBN: 979-26-0264-X, Semarang: Dian Nuswantoro University Press, pp. 98-103, 2013. 